\title{
The associating lattice gas in the presence of interacting solutes
}

Cite as: J. Chem. Phys. 142, 094502 (2015); https://doi.org/10.1063/1.4913768

Submitted: 21 August 2014 . Accepted: 18 February 2015 . Published Online: 04 March 2015

Mauricio Girardi (D), Marcia M. Szortyka, Vera B. Henriques, and Marcia C. Barbosa

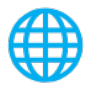

\section{ARTICLES YOU MAY BE INTERESTED IN}

Effects of confinement on anomalies and phase transitions of core-softened fluids The Journal of Chemical Physics 142, 134502 (2015); https://doi.org/10.1063/1.4916563

Lattice model for water-solute mixtures

The Journal of Chemical Physics 145, 144501 (2016); https://doi.org/10.1063/1.4964396

Single-file mobility of water-like fluid in a generalized Frenkel-Kontorova model

The Journal of Chemical Physics 147, 034510 (2017); https://doi.org/10.1063/1.4995448

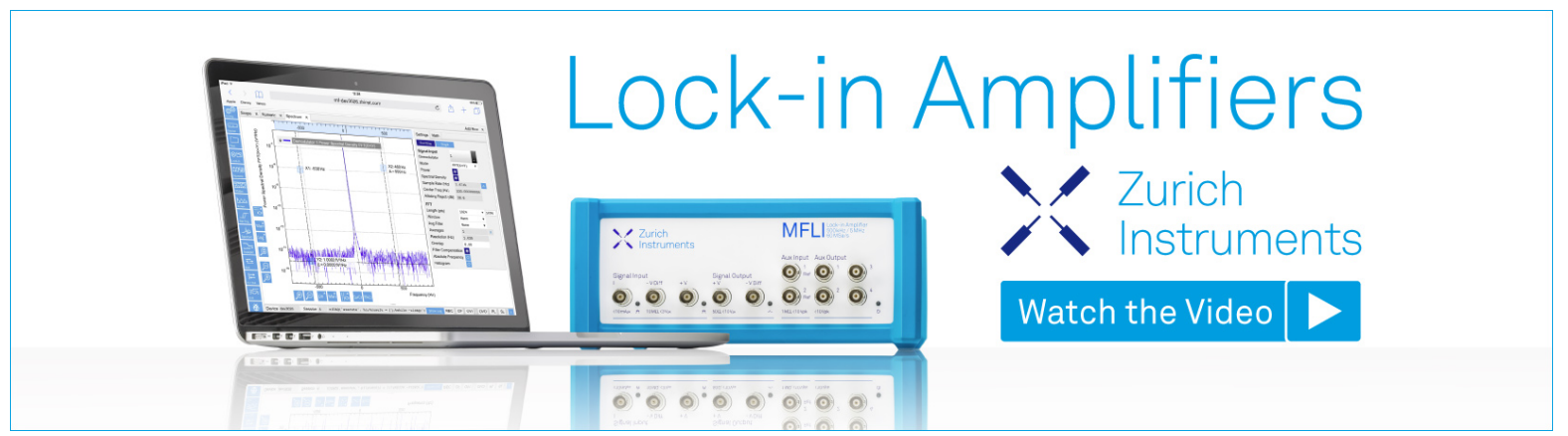




\title{
The associating lattice gas in the presence of interacting solutes
}

\author{
Mauricio Girardi, ${ }^{1, a)}$ Marcia M. Szortyka, ${ }^{1, b)}$ Vera B. Henriques, ${ }^{2, c)}$ \\ and Marcia C. Barbosa ${ }^{3, d)}$ \\ ${ }^{1}$ Universidade Federal de Santa Catarina, Rua Pedro João Pereira, 150 - Mato Alto, 88900-000 \\ Araranguá SC, Brazil \\ ${ }^{2}$ Instituto de Física, Universidade de São Paulo, Caixa Postal 66318, 05315-970 São Paulo, SP, Brazil \\ ${ }^{3}$ Instituto de Física, Universidade Federal do Rio Grande do Sul - Caixa Postal 15051, CEP 91501-970 \\ Porto Alegre, Rio Grande do Sul, Brazil
}

(Received 21 August 2014; accepted 18 February 2015; published online 4 March 2015)

\begin{abstract}
We have investigated the phase diagram of a statistical model for hydrogen-bonding solutions for polar solutes. The structured solvent is represented by an associating lattice gas, which presents anomalous density and liquid-liquid coexistence. Polar solute particles and solvent particles interact attractively, while the solvent-solvent interaction is made directional through bonding arms, which mimic hydrogen bonds. The model behavior is obtained via Monte Carlo simulations in the grandcanonical ensemble, for different sets of parameters. For small solute chemical potential and weak attraction between solute and solvent particles, addition of solute yields a shift in the transition lines of the pure solvent. This is the scenario explored by different authors, in the pursuit of stabilizing the water liquid-liquid coexistence line. However, as we show, in the case of larger solute chemical potentials, or of stronger solute-solvent attractions, new phases may arise. () 2015 AIP Publishing LLC. [http://dx.doi.org/10.1063/1.4913768]
\end{abstract}

\section{INTRODUCTION}

Most liquids contract upon cooling at any temperature range. This is not the case of anomalous fluids such as water. At ambient pressure, the specific volume of water raises if the liquid is cooled below $T \approx 4{ }^{\circ} \mathrm{C}$. The anomalous behavior of water was first suggested 300 years ago ${ }^{1}$ and was confirmed by a number of experiments. ${ }^{2,3}$ Water presents many anomalies upon cooling. For example, between $0.1 \mathrm{MPa}$ and $190 \mathrm{MPa}$ water exhibits an anomalous increasing compressibility ${ }^{4,5}$ and, at atmospheric pressure, an anomalous elevation of the isobaric heat capacity. ${ }^{6,7}$

It was proposed a few years ago that these anomalies might be related to a second critical point at the end of the coexistence line between two liquid phases, a low density liquid (LDL) and a high density liquid (HDL). ${ }^{8}$ Such a critical point was suggested from extensive molecular dynamics simulations. The numerical experiments also indicated that the critical point would be located in the supercooled region, beyond the line of homogeneous nucleation and thus could not be probed through experiments.

In order to circumvent this difficulty, a number of strategies have been considered, including confinement of the system, ${ }^{9,10}$ so that the solid structure would be suppressed. Even though quite appealing, this strategy is not out of controversy. In certain limits of confinement, the results obtained from the investigation of confined water might be quite different from the results obtained for bulk water. ${ }^{11,12}$

\footnotetext{
a)Electronic address: mauricio.girardi@ufsc.br

b) Electronic address: marcia.szortyka@ufsc.br

c) Electronic address: vhenriques@if.usp.br

d) Electronic address: marcia.barbosa@ufrgs.br
}

It has been suggested, recently, both from theoretical ${ }^{13}$ as well as from experimental studies ${ }^{14}$ that the addition of specific solutes such as glycerol ${ }^{14}$ might prevent solidification and thus allow for the observation of the liquid-liquid critical point. ${ }^{14}$ In the particular case of glycerol, the absence of the demixing transition indicates that the liquid-liquid transition observed in the mixture is not due to a solute-solvent segregation but is actually due to the solvent liquid-liquid transition. The solutes that avoid the solidification are hydrogen-bonding compounds. The basic assumption is that the addition of a small amount of species $A$, the solute, to a system with a large amount of species $B$, the solvent, yields a pressure versus temperature phase diagram with phase behavior similar to that one of the pure solvent, but with shifted coexistence lines. In the case of liquid water, the addition of specific solutes might allow for the experimental observation of the liquid-liquid phase transition, ${ }^{13-15}$ which is hindered by homogeneous nucleation in the pure solvent. This idea is based on the presumption that the interaction energy between the two species, even though attractive, as in the case of water-glycerol, is smaller than the interaction energy between particles of the solvent. The concept is quite appealing but its application to more general types of solute should be taken with a grain of salt. If the solute and solvent molecules attract each other strongly, new phases, different from the original phases of the pure solvent, may arise.

Examples of binary mixtures which may exhibit quite different phase diagrams, for different ranges of the solutesolvent interaction, are colloid-polymer mixtures ${ }^{16}$ or colloid mixtures. ${ }^{17,18}$ As the size ratio changes, these systems exhibit a very different phase diagram as compared with that of the pure colloid system. The behavior of very simple systems may also be controversial - a mixture of Lennard-Jones particles 
may show a shift in the critical properties of the pure system, ${ }^{19}$ while near the solvent liquid-gas critical point, new structures may be formed, generated by enhanced effective solute-solvent attractions..$^{20}$

In order to test which would be the conditions for the arise of the new phases, in this paper we analyze a mixture of two species, one of which is an anomalous fluid. Species $B$, the solvent, is an associated lattice gas model ${ }^{13}$ in which particles interact through a two length scales potential. The pure associated lattice gas presents a density anomaly and liquidliquid phase coexistence. In our mixture, the solute is constituted of $A$ particles which interact with each other through a hard sphere potential. As to the solute-solvent interactions, solvent particles $B$ and solute particles $A$ are attracted through a potential which mimics hydrogen bonds in the water-glycerol system. The underlying scenario we wish to explore is that of a system in which the solute-solvent interaction is more attractive than the solute-solute interaction. Solute-solute hardsphere interactions can be viewed as an extreme case of the weak solute-solute attraction. We obtain phase diagrams for different solute chemical potentials and different solvent-solute interaction constants and checked for the presence of new phases in relation to the phase diagram of the pure solvent system.

The remaining of the paper goes as follows. In Sec. II, the model is introduced and the details of the simulations are presented, and in Sec. III, results are discussed.

\section{THE MODEL}

The model solution is studied here on a body centered cubic lattice of linear size $L$. The solvent is modeled as an Associating Lattice Gas (ALG) model in three dimensions. The ALG represents an anomalous fluid with density and diffusion anomalous behavior typical of liquid water. ${ }^{21,22}$ This model follows a series of two length scales potentials that exhibit thermodynamic anomalies. ${ }^{23,24}$ The sites of a body centered cubic lattice of linear size $L$ can be occupied by a solvent molecule $\left(\sigma_{i}=1, s_{i}=0\right)$, by a solute $\left(\sigma_{i}=0, s_{i}=1\right)$ particle, or can be empty $\left(s_{i}=\sigma_{i}=0\right)$. Each solvent molecule can make up to four bonds. For that purpose, each water molecule exhibits four active arms that can be in two different configurations as seen in Fig. 1 (LDL). A bond is formed when two neighboring molecules at sites $i$ and $j$ have their arms pointing to each other. An arm is represented by orientation variable $\tau_{i j}$ that is equal to 1 when an arm of molecule at site $i$ points to site $j$, and it is 0 otherwise. ${ }^{21}$ Each time two neighbor solvent particles are bonded, the energy decreases. The polar solute molecules interact with the solvent molecules through an attractive potential. Solutes interact only through excluded volume. The energy of this system is given by

$$
\mathcal{H}=\sum_{\langle i, j\rangle}\left[\sigma_{i} \sigma_{j}\left(\epsilon+\gamma \tau_{i j} \tau_{j i}\right)+\theta \sigma_{i} s_{j}\right],
$$

where $s_{i}, \sigma_{i}=0,1$ are the solute and solvent occupational variables, respectively, $\epsilon$ is a van der Waals like energy, $\gamma$ is the bond energy, $\theta$ is the solute-solvent interaction, and $\tau_{i j}=0,1$ corresponds to the arm variable. A bond is formed when two
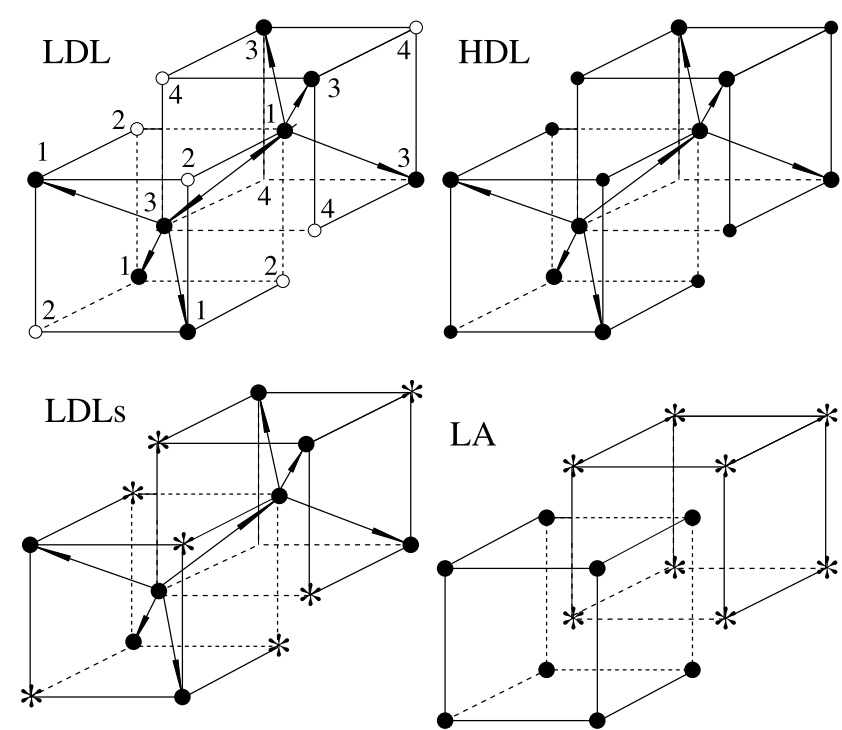

FIG. 1. Spatial representation of the $L D L, L D L s, H D L$, and $L A$ phases with water molecules (filled circles), solutes (asterisks), holes (empty circles), and $H B s$ (arrows). The numbers indicate the different sub-lattices. Note that in the $L A$ phase, sublattices 1,2 are occupied by solvent particles, while sublattices 3,4 are occupied by solute particles, forming intercalated planes.

neighboring particles have bonding arms $(\tau=1)$ pointing to each other. We choose $\epsilon>0$ and $\gamma<0$ (with $\gamma+\varepsilon<0$ ), which gives an energetic penalty on neighbors not forming a bond, and $\theta<0$ representing an effective attraction between solute and solvent.

The model we adopt here for representing water-like anomalies belongs to a large class of lattice gas models which have in common an inter-particle interaction potential with two length scales. ${ }^{23-29}$ These models show a competition between a more compact and an open structures. These two scales represent in an effective way the non-bonding and bonding interactions present in water.

Here, different solute-solvent strengths are explored with the aim to understand the effect of this interaction upon the temperature versus solvent chemical potentials phase diagram. In particular, we analyze the ranges of solute-solvent interaction strength and solute chemical potential for which a liquidliquid phase transition without demixing is present. Our results may add some qualitative understanding in relation to the experiments with low concentrations of glycerol. ${ }^{14}$

\section{A. The ground state}

At zero temperature, the grand potential per lattice site is $\Omega=e-\mu_{w} \rho_{w}-\mu_{s} \rho_{s}$, where $\rho_{w(s)}$ and $\mu_{w(s)}$ are density and chemical potential of water (solute), respectively, and $e$ $=\mathcal{H} / L^{3}$.

For very low values of the chemical potentials, the lattice is empty and the system is in the gas phase. First, let us assume that the chemical potential of the solute is negative. Starting from the gas phase and increasing the chemical potential of the solvent, the system reaches a point at which the gas phase, $\rho_{w}=\rho_{s}=0$, coexists with a liquid phase, of density $\rho_{w}=1 / 2$. This liquid phase may present different structures, depending on the value of the attractive term $\theta$ : (a) the pure $L D L$ phase 


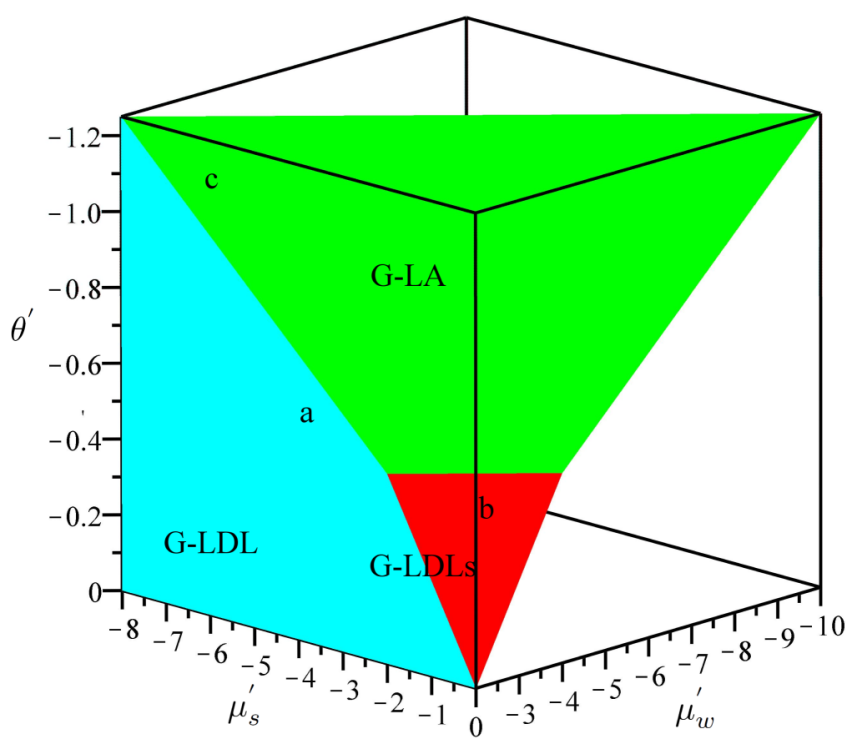

FIG. 2. Planes of phases coexistence at $T=0$ in the $\theta^{\prime}, \mu_{s}^{\prime}$, and $\mu_{w}^{\prime}$ parameter space. At higher values of the water chemical potential, the diagram is similar (but mirrored), with the $G$ phase replaced by the $H D L$ phase. Points indicate the parameter values for which the $T$ versus $\mu_{w}^{\prime}$ phase diagrams were obtained via simulations: $a$ is for $\theta^{\prime}=-0.5, \mu_{s}^{\prime}=-4$, and $\mu_{w}^{\prime}=-2 ; b$ is for $\theta^{\prime}=-0.3, \mu_{s}^{\prime}=-0.2$, and $\mu_{w}^{\prime}=-3.0 ; c$ is for $\theta^{\prime}=-1.1, \mu_{s}^{\prime}=-6$, and $\mu_{w}^{\prime}=-2.8$.

(Fig. 1), with $\left(\rho_{w}=1 / 2, \rho_{s}=0\right)$, (b) the LDLs (Low Density Liquid/Solute) phase that is the low density liquid for the solvent but with the empty sites occupied by solute particles ( $\rho_{w}=1 / 2, \rho_{s}=1 / 2$ ), and (c) the $L A$ (Alternate Layers) phase, formed by intercalated planes of sites totally filled either by pure solvent either by pure solutes $\left(\rho_{w}=1 / 2, \rho_{s}=1 / 2\right)$. In the $L A$ phase, all solutes are nearest-neighbors $(N N)$ of solvents and no water-water pair of $N N$ is present implying in a random distribution of water particle states (since no hydrogen-bond $(H B)$ is possible).

We may write expressions for the grand potential per number of lattice sites, $\Omega$, for the gas phase and for each of the three possible low density liquid phases,

$$
\begin{aligned}
\Omega_{G} & =0, \\
\Omega_{L D L} & =\varepsilon+\gamma-\frac{\mu_{w}}{2}, \\
\Omega_{L D L s} & =\Omega_{L D L}+\left(2 \theta-\frac{\mu_{s}}{2}\right), \\
\Omega_{L A} & =4 \theta-\frac{\mu_{w}}{2}-\frac{\mu_{s}}{2} .
\end{aligned}
$$

At low values of the water chemical potential, different phases may coexist depending on the values of

$$
\begin{aligned}
\theta^{\prime} & =\frac{\theta}{|\varepsilon+\gamma|}, \\
\mu_{s}^{\prime} & =\frac{\mu_{s}}{|\varepsilon+\gamma|}, \\
\mu_{w}^{\prime} & =\frac{\mu_{w}}{|\varepsilon+\gamma|}
\end{aligned}
$$

(note that $\varepsilon+\gamma<0$ here). Fig. 2 exhibits the coexistence planes $(G-L D L, G-L D L s$, and $G-L A)$ in the $\theta^{\prime}, \mu_{s}^{\prime}$, and $\mu_{w}^{\prime}$ parameter space. The lines of intersection of the twophase coexistence planes represent three-phase coexistence, while the point at which the three lines cross indicates fourphase coexistence. In Table I, we indicate the ranges of model parameters $\theta^{\prime}, \mu_{s}^{\prime}$, and $\mu_{w}^{\prime}$ for which three- and four-phase coexistence is present.

As the solvent chemical potential increases, the low density liquid phases stabilize. For even higher solvent chemical potentials, a new liquid phase appears, the $H D L$. This phase coexists with the low density liquid, which may be $L A, L D L$, or $L D L s$, depending on the value of $\theta$.

The specific geometry of the $L A$ depends on the lattice structure. In the $A L G$ lattice, there is the formation of layers. In other geometries, other types of intercalating structures would be formed.

For the $H D L$ phase (Fig. 1), the model grand-potential is given by

$$
\Omega_{H D L}=4 \varepsilon+2 \gamma-\mu_{w} .
$$

This yields a ground state phase diagram for high solvent chemical potential which presents analogous geometry (but with mirrored planes) to the phase diagram of Fig. 2, for low solvent chemical potentials, if one replaces $G$ with $H D L$. The ranges for the $\theta^{\prime}, \mu_{s}^{\prime}$, and $\mu_{w}^{\prime}$ parameters which yield three and four phase coexistence which includes the $H D L$ phase are presented in Table I.

For positive values of the solute chemical potential and negative values of the solvent chemical potential, no gas phase is present. Instead, a pure solute $S$ phase appears with $\Omega_{S}$ $=-\mu_{s}$. As the chemical potential of the solvent increases, new phase coexistences appear. At $\mu_{s}=0$, the $G$ and $S$ phases can coexist with $L D L, L D L s$, or $L A$. The $\mu_{s} \geq 0$ regime implies a solute dominated system. This case will not be analyzed here.

TABLE I. Ranges of parameters $\theta^{\prime}, \mu_{s}^{\prime}$, and $\mu_{w}^{\prime}$ for which 3- and 4-phase coexistence is present. Here, $c=(4 \varepsilon+2 \gamma) /|\varepsilon+\gamma|$ and $(\varepsilon+\gamma)<0$.

\begin{tabular}{llll}
\hline \hline 3-phases & & & \\
\hline$G$ - $L D L-L A$ & $\theta^{\prime}<-\frac{1}{2}$ & $\mu_{s}^{\prime}=8 \theta^{\prime}+2$ & $\mu_{w}^{\prime}=-2$ \\
$G$ - $L D L-L D L s$ & $-\frac{1}{2}<\theta^{\prime}<0$ & $\mu_{s}^{\prime}=4 \theta^{\prime}$ & $\mu_{w}^{\prime}=-2$ \\
$G$ - LDLs-LA & $\theta^{\prime}=-\frac{1}{2}$ & $-2<\mu_{s}^{\prime}<0$ & $\mu_{w}^{\prime}=-4-\mu_{s}^{\prime}$ \\
$H D L-L D L-L A$ & $\theta^{\prime}<-\frac{1}{2}$ & $\mu_{s}^{\prime}=8 \theta^{\prime}+2$ & $\mu_{w}^{\prime}=2 c+2$ \\
$H D L-L D L-L D L s$ & $-\frac{1}{2}<\theta^{\prime}<0$ & $\mu_{s}^{\prime}=4 \theta^{\prime}$ & $\mu_{w}^{\prime}=2 c+2$ \\
$H D L-L D L s-L A$ & $\theta^{\prime}=-\frac{1}{2}$ & $-2<\mu_{s}^{\prime}<0$ & $\mu_{w}^{\prime}=2 c+4+\mu_{s}^{\prime}$ \\
4-phases & & & \\
\hline$G-L D L-L D L s-L A$ & $\theta^{\prime}=-\frac{1}{2}$ & $\mu_{s}^{\prime}=-2$ & $\mu_{w}^{\prime}=-2$ \\
$H D L-L D L-L D L s-L A$ & $\theta^{\prime}=-\frac{1}{2}$ & $\mu_{s}^{\prime}=-2$ & $\mu_{w}^{\prime}=2 c+2$ \\
\hline \hline
\end{tabular}


In previous work, we have explored the $c=2 \epsilon+4 \gamma=0$ for pure solvent ${ }^{30}$ and for a fixed small concentration of solute. ${ }^{22}$ In this case, $\theta^{\prime}=-0.3$ and $\mu_{s}=-0.2$ lead to a $G-L D L s$ transition at $\mu_{w}^{\prime}=-3.0$ as illustrated in the Figure 2 and to a $L D L s-H D L$ with $\mu_{w}^{\prime}=3.0$.

The case in which $\mu_{s}=4 \theta$ is also interesting. For this value of the solute chemical potential the LDL and LDLs phases coexist. However, this coexistence is only stable for $2+2 c>\mu_{w}^{\prime}>-2$ and $0>\mu_{s}^{\prime}>-2$, otherwise the gas, HDL, or the LA phases are stable.

\section{B. Simulation details}

For finite temperatures, we have investigated our model system through Monte Carlo simulations in the grand canonical ensemble. The solvent and solute chemical potentials together with the temperature are kept fixed, allowing for the total number of particles to fluctuate. A typical simulation run can be summarized as follows. The lattice with $V=L^{3}$, for $L=20$, starts completely empty and the temperature and chemical potentials are fixed. In one Monte Carlo step $(M C s)$, each lattice site is tested sequentially for insertion or removal of either a water molecule or a solute particle. If the site is empty, we try to insert either a solute particle (with probability $1 / 3$ ) or a water particle (with probability $2 / 3$ ), with one of the two possible states randomly chosen. The insertion/removal of a water (solute) molecule is then tested with the usual Metropolis prescription, namely,

$$
P_{w(s)}=\left\{\begin{array}{ll}
1, & \Delta \mathcal{H}-\mu_{w(s)} \leq 0 \\
\frac{1}{3} \exp \left[-\left\{\Delta \mathcal{H}-\mu_{w(s)}\right\} / k_{B} T\right], & \Delta \mathcal{H}-\mu_{w(s)}>0
\end{array},\right.
$$

where $k_{B}$ is Boltzmann's constant and $T$ is the temperature. After the insertion/removal step, each water molecule is allowed to exchange its state with probability $P=\min (1, \exp [-\Delta \mathcal{H} /$ $\left.k_{B} T\right]$ ). The system is then thermalized, the quantities of interest are measured, and their mean-values calculated.

\section{RESULTS}

In this section, the solvent chemical potential versus temperature phase diagrams are presented and discussed. Data were obtained from Monte Carlo simulations for the three different regions of the space of parameters $\theta^{\prime}$ and $\mu_{s}^{\prime}$ of Fig. 2 (the temperature given in units of $|\varepsilon+\gamma| / k_{B}$ ).

For very low values of $\mu_{s}^{\prime}$, the solute appears in small amounts. For instance, in the case of $\theta^{\prime}=-0.5$ and $\mu_{s}^{\prime}=-4$ (point $a$ in Fig. 2), the solvent chemical potential versus temperature phase diagram is very similar to that of the pure liquid, as can be seen in Fig. 3. The weak water-solute interaction, small solute chemical potential, and the high mixing entropy lead to a low solute concentration, and critical line temperatures are shifted to lower values when compared with the pure solvent case. The temperature of maximum density (TMD), which represents the loci where the solvent is denser, also maintains the same general behavior in spite of the shift to lower values.

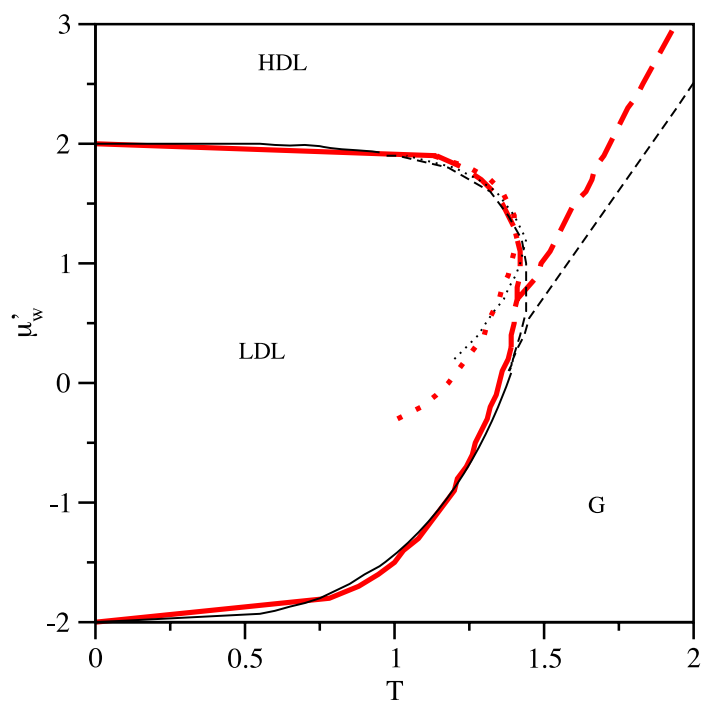

FIG. 3. Phase diagram for the solution, in the $T$ versus $\mu_{w}^{\prime}$ plane, for $\theta^{\prime}=-0.5$ and $\mu_{s}^{\prime}=-4$ (thick-red lines). Pure solvent transition lines are also shown (thin-black lines). Here, continuous lines are coexistence lines, dashed lines are critical lines, and the dotted lines indicate the TMD.

For higher values of the solute chemical potential, but intermediate values of the solute-solvent interaction potential $\theta^{\prime}$, the solute fills the empty sites and the system reaches the region of the solvent chemical potential $T=0$ phase diagram in which the gas phase coexists with the LDLs, phase, as illustrated in Fig. 2 (point $b$ ). In the LDLs phase, at $T=0$, half of the lattice is filled with the solute particles and half with the solvent particles. The solvent chemical potential versus temperature phase diagram for $\theta^{\prime}=-0.3$ and $\mu_{s}^{\prime}=-0.2$ is shown in Fig. 4 . The pure solvent case is illustrated for comparison. As can be seen, at low temperatures, the $L D L s$ phase covers a larger area of the $\mu_{w}^{\prime}$ values, if compared with the $L D L$ of the pure solvent phase, illustrating that the solvent-solute interaction plays an important role. However, the topology of the diagrams remains

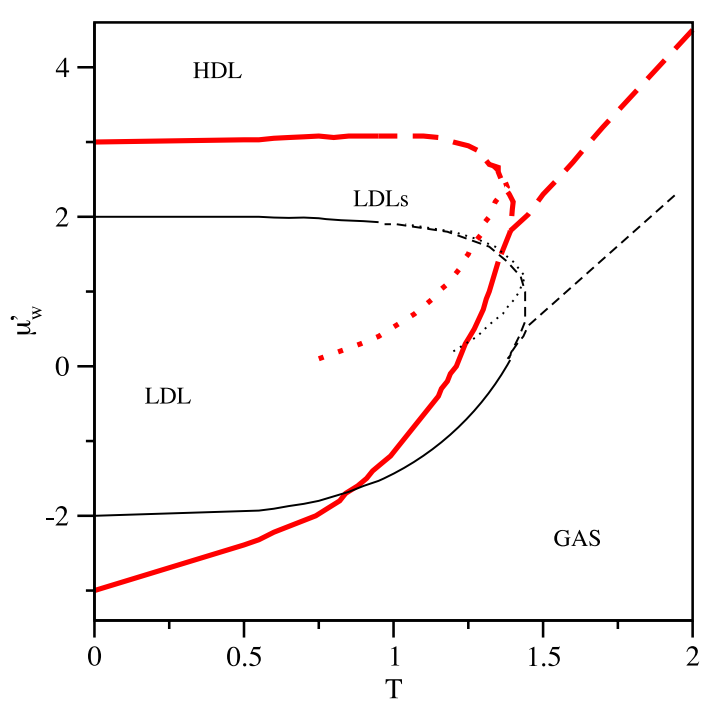

FIG. 4. Phase diagram in the $T$ versus $\mu_{w}^{\prime}$ plane, for $\theta^{\prime}=-0.3$ and $\mu_{s}^{\prime}=-0.2$ (thick-red lines). Pure solvent transition lines are also displayed (thin-black lines). Here, continuous lines are coexistence lines, dashed lines are critical lines, and the dotted lines are the TMD. 
almost the same. In this solvent chemical potential versus temperature phase diagram, the $H D L$ phase has a mixture of solute and solvent distributed in such a way as to maximize the water-water bonds.

Another important result shown in this diagram is that, in a major portion of it, the liquid-gas transition line is shifted to lower temperatures. This is expected for non-ideal solutions for which the solvent-solute interaction is weaker than the solvent-solvent potential, since the enthalpy change for mixing is negative. ${ }^{31}$ The shifts shown in this case are consistent with mixtures of water with polar and ionic solutes. ${ }^{13,15}$

At the liquid-liquid $L D L s-H D L$ transition, the nature of the coexistence and critical lines is the same as for the transitions observed in the pure solvent case. As in the case of glycerol, no demixing is observed. ${ }^{14}$ Due to the solvent-solute interaction, the two species are mixed in both phases.

For larger values of solute $=$ solvent interaction $\theta^{\prime}$ and larger values of $\mu_{s}^{\prime}$, the gas phase coexists with the $L A$ phase at $T=0$, as shown in Fig. 2 (point $c$ ). The number of solutesolvent molecules which are nearest-neighbors increases and planes of solvent alternate with planes of solute, in the alternate liquid phase ( $L A$ in Fig. 1). The energetic penalty for breaking water-water bonds is compensated with the maximized watersolute interactions and the high water entropy (solvents are in random orientational states when forming planes). The phase diagram for $\theta^{\prime}=-1.1$ and $\mu_{s}^{\prime}=-6$ is shown in Fig. 5, and the change in topology is quiet striking, if compared to that of pure solvent. The liquid-gas transition line is shifted to higher temperatures, which is again expected for non-ideal solutions with strong solute-solvent interactions. It is also evident that a new phase not present in the pure solvent scenario appears. At higher temperatures, the $L A$ phase is separated from the fluid phase by a critical line. This result is consistent with hard sphere mixtures, for which, depending on the size of the particles, the solution exhibits phases not present in the pure system..$^{17,18}$

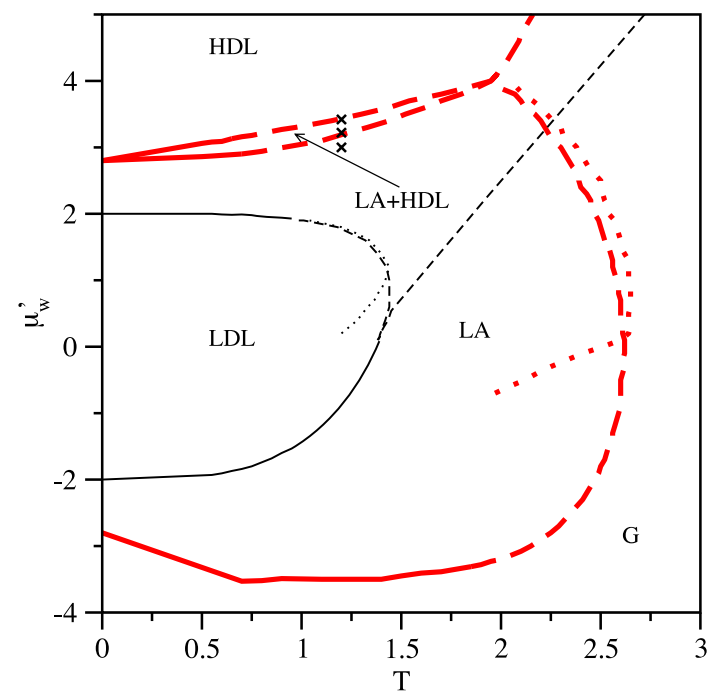

FIG. 5. Phase diagram in the $T$ versus $\mu_{w}^{\prime}$ plane, for $\theta^{\prime}=-1.1$ and $\mu_{s}^{\prime}=-6$ (thick-red lines). Pure solvent transition lines are also shown (thin-black lines). Here, continuous lines are coexistence lines, dashed lines are critical lines, and the dotted lines are the TMD. Crosses are the points explored in Fig. 10.

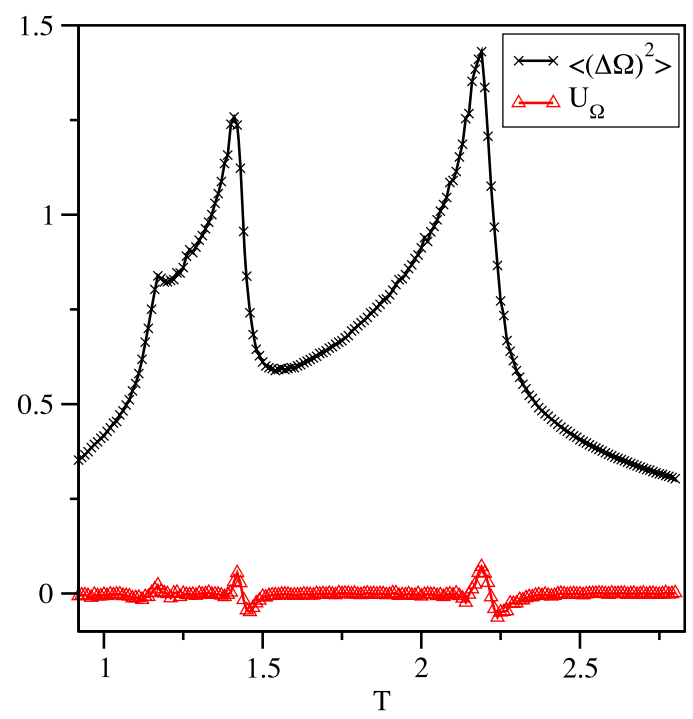

FIG. 6. Fluctuation of energy $\Omega\left\langle(\Delta \Omega)^{2}\right\rangle_{M C s}$ and its cumulant $U_{\Omega}$ as a function of temperature for $\theta^{\prime}=-1.1, \mu_{s}^{\prime}=-6$, and $\mu_{w}^{\prime}=3.4$. The peaks indicate the crossing of transition lines in the phase diagram of Fig. 5.

At very high values of the solvent chemical potential, the $H D L$ phase appears. Since the solvent and solute chemical potentials and the solvent-solute interaction term are large, the $H D L$ phase has a mixture of solvent and solute. The high value of $\theta^{\prime}$ leads to a loss of structure in the $H D L$ phase and solute and solvent molecules are randomly distributed.

At zero temperature, the $H D L$ and the $L A$ coexist at $\mu_{w}^{\prime}$ $=2.8$ for $\mu_{s}^{\prime}=-6$ and $\theta^{\prime}=-1.1$. As temperature is increased, the boundary between the two phases is not well defined. It is a coexistence line for low temperatures but as the temperature is increased two critical transitions appear. In order to clarify this point, additional methods were employed. Fig. 6 exhibits the behavior of the fluctuations of $\Omega=e-\mu_{w} \rho_{w}-\mu_{s} \rho_{s}$ and its cumulant as a function of temperature, for $\theta^{\prime}=-1.1$.

As the temperature is lowered (at constant solvent chemical potential), three different transition lines are crossed as shows the three peaks in $\left\langle(\Delta \Omega)^{2}\right\rangle_{M C s}$ : (1) fluid- $L A$, (2) $L A$ $L A+H D L$, and (3) $L A+H D L-H D L$ transitions. The peak at $T \approx 2.2$ is related to the $L A$-fluid critical line and the two peaks at $T \approx 1.4$ and $T \approx 1.16$ are two critical lines. The critical line at $T \approx 1.4$ separates the $L A$ from a phase in which the system exhibits clusters of molecules in the $L A$ phase and fractions at $H D L$ phase $(L A-L A+H D L)$. The critical line at $T \approx 1.16$ separates this $L A-H D L$ coexistence phase from the pure $H D L$ phase. These two lines are continuous lines for $T>0.6$. At $T<0.6$, "freezing" of the system prevents us from the possibility of establishing the order of the transitions.

The $\Omega=e-\mu_{w} \rho_{w}-\mu_{s} \rho_{s}$ cumulant illustrated for two different system sizes in Fig. 7 suggests that the $G-L A$, the $L A-L A+H D L$, and the $L A+H D L-H D L$ are continuous transitions for $\mu_{s}^{\prime}=-6$ and $\mu_{w}^{\prime}=3.4$.

Fig. 1 illustrates four sublattices $i=1, \ldots, 4$ with densities $\rho_{w}^{i}$. In the gas phase, the four sublattices are equally populated in the states 1 and 2. In the transition from the gas to the $L D L s$ phase, the sublattices $i=1$ become occupied in the state 1 and the sublattice 3 in the state 2 (or vice-versa), while the sublattices $i=2,4$ become empty. In the case of the transition 


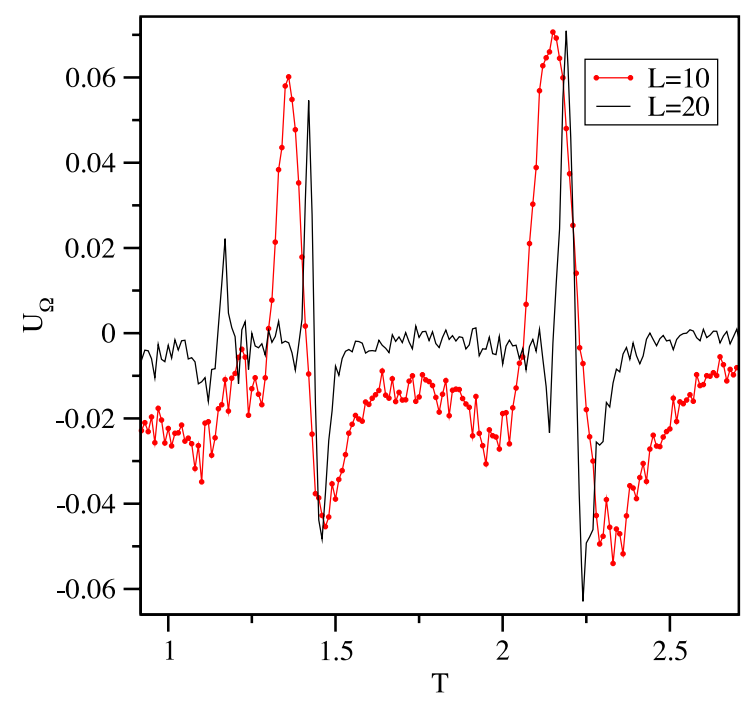

FIG. 7. Cumulant of energy $U_{\Omega}$ as a function of temperature for two lattice sizes and $\theta^{\prime}=-1.1, \mu_{s}^{\prime}=-6$, and $\mu_{w}^{\prime}=3.4$.

from the gas to the $L A$ phases, the sublattices $i=1,2$ become occupied, while the sublattices $i=3,4$ are occupied by solute. Here, the sites are occupied in a random distribution of the states 1 and 2 . In the HDL phase, all sublattices become occupied, with sublattices 1,2 in the state 1 while the sublattices 3,4 in the state 2 (or vice-versa).

The transitions from the gas to the $L A$ phase, then from the $L A$ phase to the $L A+H D L$ mixed phase, and from this to the $H D L$ are illustrated in the Fig. 8(a). The figure shows for $\theta^{\prime}=-1.1, \mu_{s}^{\prime}=-6$, and $\mu_{w}^{\prime}=3.4$ the number density

$$
\rho_{w}^{i}=\frac{\left|\left\langle n^{i}\right\rangle\right|_{w}}{n_{t}}
$$

versus temperature, where $n^{i}$ is the number of solvent particles at the sublattice $i=1, \ldots, 4$, while $n_{t}$ is the total number of solvent particles. The $L A+H D L$ region in the graph is restricted by the two dashed vertical lines.

a)

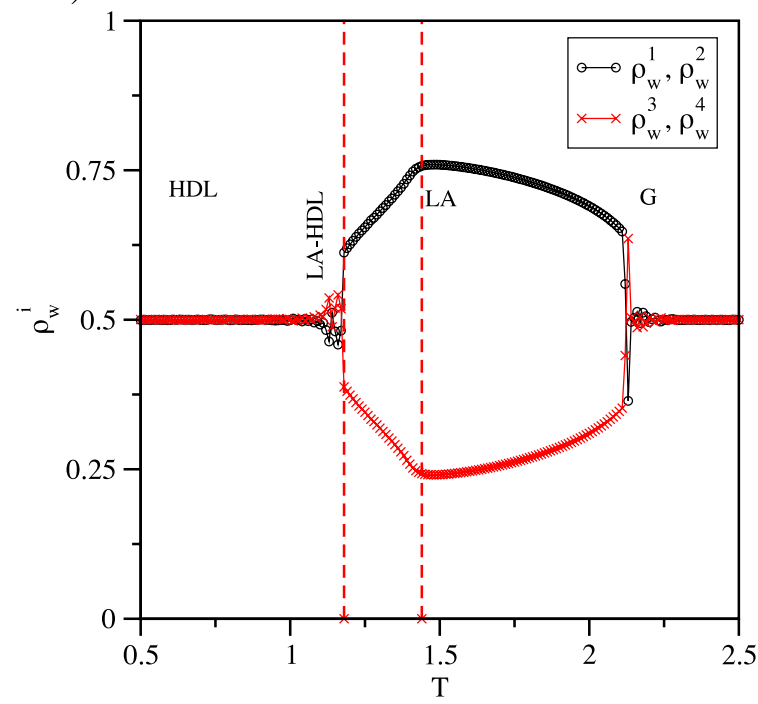

In addition to the sublattice density of solvent, another interesting quantity to understand the different phases is a directional order parameter $\delta_{x}^{i}$,

$$
\delta_{x}^{i}=\frac{\left\langle N_{x}^{i}\right\rangle}{n_{t}},
$$

where $i=1, \ldots, 4$ is the sublattice index, $N_{x}^{i}$ is the number of solvent particles in the orientational state $x=1,2$ in the sublattice $i=1, \ldots, 4$, and $n_{t}$ is the total number of solvent particles. Figure 8(b) shows $\delta_{1}^{1}+\delta_{1}^{2}$ and $\delta_{1}^{3}+\delta_{1}^{4}$ versus temperature for $\mu_{w}^{\prime}=3.4$.

In $L A$ phase, $\rho_{w}^{1}=\rho_{w}^{2} \rightarrow 1 / 2$ while $\rho_{w}^{3}=\rho_{w}^{4} \rightarrow 0$ as $T$ $\rightarrow 0$. Since in the $L A$ phase both 1 and 2 states are equally probable, $\delta_{1}^{1}+\delta_{1}^{2} \rightarrow 1 / 2$ as $T \rightarrow 0$. In addition, $\delta_{1}^{3}+\delta_{1}^{4} \rightarrow 0$ simply because the sublattices become occupied by solute.

In the $H D L, \rho_{w}^{1}=\rho_{w}^{2}=\rho_{w}^{3}=\rho_{w}^{4} \rightarrow 1 / 4$ as $T \rightarrow 0$. In the $H D L$ phase, the sublattices 1,2 are in the state 1 , while the sublattices 3,4 are in the state 2 (or vice-versa), therefore, $\delta_{1}^{1}+\delta_{1}^{2} \rightarrow 1 / 2$ while $\delta_{1}^{3}+\delta_{1}^{4} \rightarrow 0$ (or vice-versa)

The $G-L A$ and the $L A+H D L-H D L$ densities illustrated in Fig. 8(a) show an abrupt change that could indicate a first order transition. For the $L A+H D L-H D L$ transition, the dependence of $\delta_{1}^{1}+\delta_{1}^{2}$ with the temperature suggests a continuous transition. This result is consistent with the cumulant of $\Omega=e$ $-\mu_{w} \rho_{w}-\mu_{s} \rho_{s}$ illustrated in Fig. 7.

In the $L A-L A+H D L$ region, both the density in Fig. 8(a) and the $\delta_{1}^{1}+\delta_{1}^{2}$ in Fig. 8(b) do not indicate the presence of a phase transition. The cumulant of $\Omega=e-\mu_{w} \rho_{w}-\mu_{s} \rho_{s}$, however, illustrated in Fig. 7 shows the presence of a continuous phase transition. The presence of two possible orientations $\delta_{1}^{1}+\delta_{1}^{2} \rightarrow 1 / 2$ while $\delta_{1}^{3}+\delta_{1}^{4} \rightarrow 0$ or $\delta_{1}^{1}+\delta_{1}^{2} \rightarrow 0$ while $\delta_{1}^{3}$ $+\delta_{1}^{4} \rightarrow 1 / 2$ at the $H D L$ implies that even at the $L A$ phase there are more than one possibility of ordering leading to the fluctuations observed in the Fig. 8(b).

Another confirmation for the presence of a continuous phase transition between the $L A-L A+H D L$ phase comes from the order parameter $\phi$ that measures the degree of orientational

b)

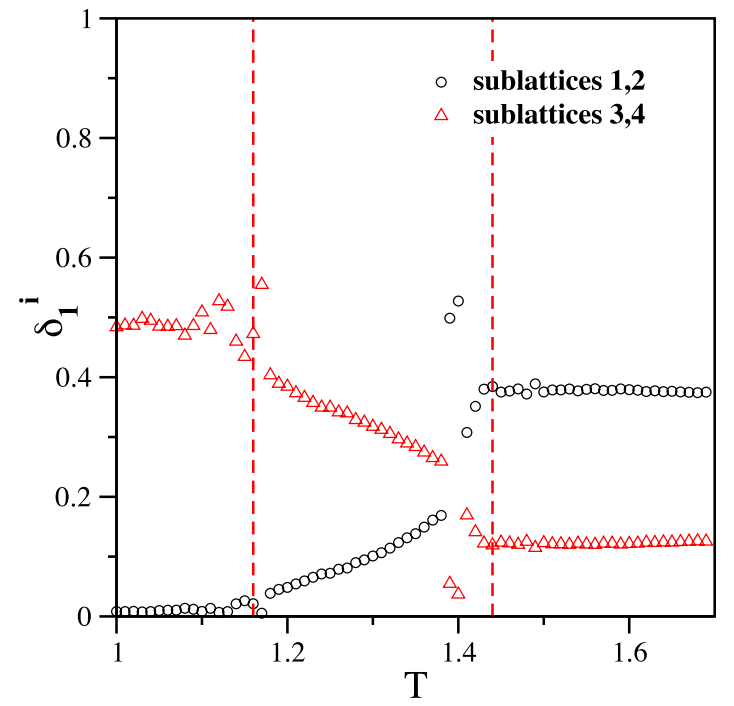

FIG. 8. (a) Fraction of total water $\rho_{w}^{i}$ in sublattice $i$ and (b) orientational order parameter $\delta_{x}^{i}$ for $x=1$ as a function of temperature. Here, $\theta^{\prime}=-1.1, \mu_{s}^{\prime}=-6$, and $\mu_{w}^{\prime}=3.4$. The dashed vertical lines delimit the $L A+H D L$ coexistence region. 


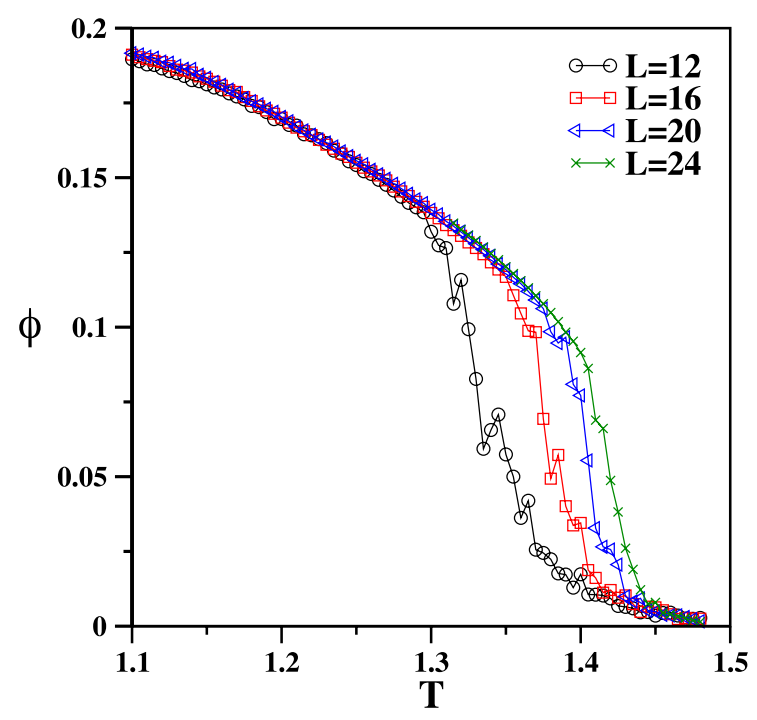

FIG. 9. Order parameter $\phi$ versus temperature for $L=12,16,20,24$ and $\mu_{w}^{\prime}=3.4$ crossing the $L A-L A+H D L$ phase separation.

order of water molecules as defined by Ref. 32 given, in our case, by

$$
\phi=\left|\sum_{i=1}^{4} \frac{\left(N_{1}^{i}-N_{2}^{i}\right)}{4 n_{t}}\right| .
$$

This parameter is illustrated in Fig. 9 versus temperature for the reduced chemical potential, $\mu_{w}^{\prime}=3.4$ for different lattice sizes $L=12,16,20,24$. The smooth increase of $\phi$ at $T$ $\approx 1.4$ suggests a continuous phase transition.

The solute cluster size distribution was also investigated in order to locate the transition lines of the $L A+H D L$ region. In Fig. 10, the volume fraction $f(i)$ of solute clusters of size $i$ is plotted at $T=1.2$ and for three different values of $\mu_{w}^{\prime}$. For $\mu_{w}^{\prime}$ inside the $L A$ phase, the system exhibits a typical cluster size leading to a maximum in $f\left(i_{\max }\right)$. These clusters of solutes appear as a result from the thermal fluctuations of the intercalated layers, since in the pure $L A$, no water or solute

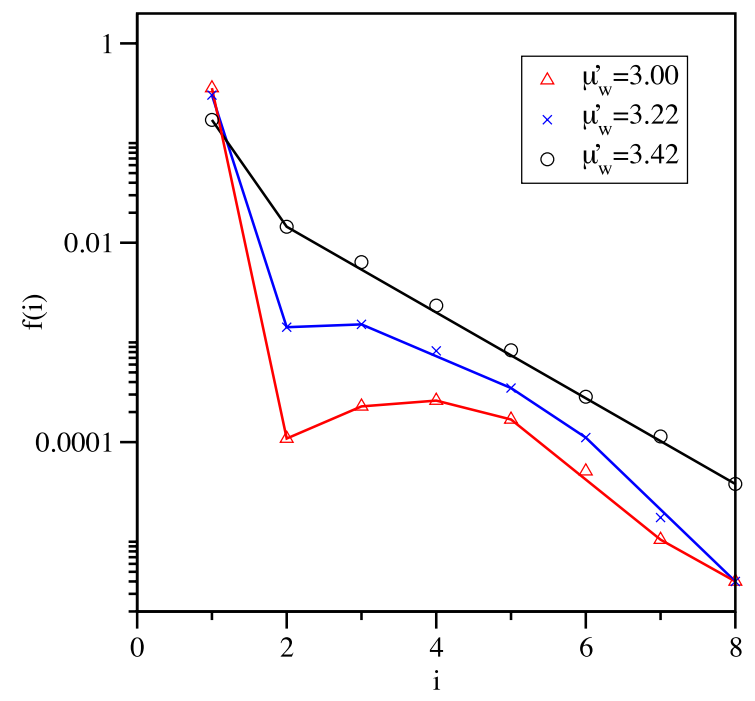

FIG. 10. Volume fraction $f(i)$ of solute clusters of size $i$ for $\theta^{\prime}=-1.1$, $\mu_{s}^{\prime}=-6, T=1.2$ and three different values of $\mu_{w}^{\prime}$. cluster is present (no pair is of $N N \mathrm{~s}$ ). As we approach the lower line, this typical cluster disappears and $f(i)$ turns to be monotonic. This indicates an ideal gas behavior of solute in the structured $H D L$ solvent at higher values of $\mu_{w}^{\prime}$. The locus of the lower transition line is then estimated by the change in the $f(i)$ behavior which, in this case, occurs for $\mu_{w}^{\prime}=3.22$, well in accordance with $U_{\Omega}$ estimate.

\section{CONCLUSIONS}

In this paper, we have addressed the question if the addition of solute to an anomalous liquid leads to the appearance of new phases or just shifts the values of temperature and chemical potential of the pure solvent phase space.

For that purpose, the chemical potential versus temperature phase diagram of solute plus solvent was explored.

If either the solute-solvent interaction or the solute chemical potential is high enough, new phases, $L A$ and $L D L s$, respectively, appear. In the first case, a strong solvent-solute interaction yields layering of the system ( $L A$ phase) in which solute particles form layers intercalated with solvent. In this case, since no water-water pair is present, each solvent can be in a random state, leading to a residual entropy per particle $s$ $=k_{B} \ln 2$.

For strong solute-solvent interaction $\left(\theta^{\prime}\right)$, the finite temperature behavior reveals a change in the phase diagram topology, if compared to that of the pure fluid. Not only is the $L D L$ replaced by the $L A$ phase, but also a $L A+H D L$ mixed phase is present before the transition to the HDL phase. This narrow stripe in the phase diagram is delimited by continuous transition lines at high temperature. These lines join two other coexistence lines, or a single point, which cannot be confirmed yet by the current simulation data.

We have shown that in addition to a shift in the temperature of the critical lines, the addition of a very hydrophilic solute might lead to the appearance of new phases.

\section{ACKNOWLEDGMENTS}

This work is partially supported by CNPq, Capes, INCTFCx, Universidade Federal de Santa Catarina, and Universidade de São Paulo.

${ }^{1}$ R. Waler, Essays of Natural Experiments (Johnson Reprint, New York, 1964).

${ }^{2}$ G. S. Kell, J. Chem. Eng. Data 20, 97 (1975).

${ }^{3}$ C. A. Angell, E. D. Finch, and P. Bach, J. Chem. Phys. 65, 3063 (1976).

${ }^{4}$ R. J. Speedy and C. A. Angell, J. Chem. Phys. 65, 851 (1976).

${ }^{5}$ H. Kanno and C. A. Angell, J. Chem. Phys. 70, 4008 (1979).

${ }^{6}$ C. A. Angell, M. Oguni, and W. J. Sichina, J. Phys. Chem. 86, 998 (1982).

${ }^{7}$ E. Tombari, C. Ferrari, and G. Salvetti, Chem. Phys. Lett. 300, 749 (1999).

${ }^{8}$ P. H. Poole, F. Sciortino, U. Essmann, and H. E. Stanley, Nature (London) 360, 324 (1992).

${ }^{9}$ L. Liu, S.-H. Chen, A. Faraone, S.-W. Yen, and C.-Y. Mou, Phys. Rev. Lett. 95, 117802 (2005).

${ }^{10}$ F. Mallamace, C. Branca, M. Broccio, C. Corsaro, N. Gonzalez-Segredo, J. Spooren, H. E. Stanley, and S. H. Chen, Eur. Phys. J.: Spec. Top. 161, 19 (2008).

${ }^{11}$ N. Giovambattista, P. J. Rossky, and P. G. Debenedetti, Phys. Rev. E 73, 041604 (2006)

${ }^{12}$ M. M. Szortyka, M. Girardi, V. Henriques, and M. C. Barbosa, J. Chem. Phys. 130, 094504 (2009). 
${ }^{13}$ J. W. Biddle, V. Holten, and M. A. Anisimov, J. Chem. Phys. 141, 074504 (2014).

${ }^{14}$ K. Murata and H. Tanaka, Nat. Commun. 4, 2844 (2013).

${ }^{15}$ D. Corradini, M. Rovere, and P. Gallo, J. Phys. Chem. B 115, 1461 (2011).

${ }^{16}$ H. N. W. Lekkerkerker, W. C.-K. Poon, P. N. Pusey, A. Stroobants, and P. B. Warren, Europhys. Lett. 20, 559 (1992).

${ }^{17}$ A. Imhof and J. K. G. Dhont, Phys. Rev. Lett. 75, 1662 (1995).

${ }^{18}$ M. Dijkstra, R. van Roij, and R. Evans, Phys. Rev. E 59, 5744 (1999).

${ }^{19}$ J. J. Potoff and A. Z. Panagiotopoulos, J. Chem. Phys. 109, 10914 (1998).

${ }^{20}$ I. B. Petsche and P. G. Debenedetti, J. Chem. Phys. 91, 7075 (1989).

${ }^{21}$ M. Girardi, A. L. Balladares, V. Henriques, and M. C. Barbosa, J. Chem. Phys. 126, 064503 (2007).

${ }^{22}$ M. Szortyka, M. Girardi, V. B. Henriques, and M. C. Barbosa, J. Chem. Phys. 137, 064905 (2012).

${ }^{23}$ N. G. Almarza, J. A. Capitan, J. A. Cuesta, and E. Lomba, J. Chem. Phys. 131, 124506 (2009).
${ }^{24}$ C. Buzano and M. Pretti, J. Chem. Phys. 119, 3791 (2003).

${ }^{25}$ S. Sastry, P. G. Debenedetti, and F. Sciortino, Phys. Rev. E 53, 6144 (1996).

${ }^{26}$ C. Buzano, E. De Stefanis, A. Pelizzola, and M. Pretti, Phys. Rev. E 69, 061502 (2004).

${ }^{27}$ G. Franzese, M. I. Marques, and H. E. Stanley, Phys. Rev. E 67, 011103 (2003).

${ }^{28}$ A. L. Balladares, M. V. Henriques, and M. C. Barbosa, J. Phys.: Condens. Matter 19, 116105 (2009).

${ }^{29}$ C. J. Roberts and P. G. Debenedetti, J. Chem. Phys. 105, 658 (1996).

${ }^{30}$ M. M. Szortyka, M. Girardi, V. Henriques, and M. C. Barbosa, J. Chem. Phys. 132, 134904 (2010)

${ }^{31}$ R. H. Petrucci, F. G. Herring, J. D. Madura, and C. Bissonnette, General Chemistry, 10th ed. (Pearson Canada, Toronto, 2007).

${ }^{32}$ C. Buzano, E. De Stefanis, and M. Pretti, J. Chem. Phys. 129, 024506 (2008). 